\title{
Comparison of the spatial-frequency selectivity of local and global motion detectors
}

\author{
Peter J. Bex and Steven C. Dakin \\ Institute of Ophthalmology, 11-43 Bath Street, London EC1V 9EL, UK
}

Received April 6, 2001; accepted August 8, 2001; revised manuscript received September 14, 2001

\begin{abstract}
Convergent physiological and behavioral evidence indicates that the initial receptive fields responsible for motion detection are spatially localized. Consequently, the perception of global patterns of movement (such as expansion) requires that the output of these local mechanisms be integrated across visual space. We have differentiated local and global motion processes, with mixtures of coherent and incoherent moving patterns composed of bandpass filtered dots, and have measured their spatial-frequency selectivity. We report that local motion detectors show narrow-band spatial-frequency tuning (i.e., they respond only to a narrow range of spatial frequencies) but that global motion detectors show broadband spatial-frequency tuning (i.e., they integrate across a broad range of spatial frequencies), with a preference for low spatial frequencies. () $2002 \mathrm{Op}$ tical Society of America

OCIS codes: $330.0330,330.6110$.
\end{abstract}

\section{INTRODUCTION}

Physiological $^{1-3}$ and behavioral ${ }^{4}$ studies have shown that the receptive fields of early visual mechanisms are highly selective for a limited range of stimulus attributes, such as spatial frequency and direction of movement, and that they are relatively small, responding to structure within only a very limited region of the visual field. However, the visual system is required to process information about a variety of motions, such as ego motion or the movements of large objects, which cannot be derived directly from such mechanisms and must be the result of combining a number of independent, local detectors across visual space. Such a view is consistent with a variety of recent behavioral $^{5-17}$ and electrophysiological ${ }^{18-25}$ studies proposing that local motion signals form only the first stage of a hierarchically organized system that specializes in more complex global pattern motion at later stages.

Several investigators have examined the selectivity of motion detection mechanisms in humans with moving patterns of limited spatial-frequency content. Many studies have focused on estimating $d_{\max }$, the largest abrupt displacement of local features that observers can detect before their movement appears incoherent, ${ }^{26}$ and it is now well established that $d_{\text {max }}$ scales inversely with the spatial frequency of narrow-band patterns. ${ }^{27-35}$ Furthermore, the spatial-frequency structure of a moving pattern must be similar across each displacement to support motion detection ${ }^{36}$ or to prevent the occurrence of phi motion $^{37}$ (illusory motion in the opposite direction), implying that motion detectors are narrowly tuned to the spatial frequency of their input. ${ }^{38}$ However, motion perception can be masked by a broad range of spatial frequencies, ${ }^{39}$ which instead implicates broad spatialfrequency selectivity for motion detectors.

A similar discrepancy in estimates of the spatialfrequency tuning of motion detectors has been found in studies of the motion aftereffect. With static test patterns, the longest motion aftereffect is elicited when the adapting and test gratings are of similar spatial frequency, ${ }^{40-43}$ implying narrow spatial-frequency tuning. However, with counterphase flickering test patterns (at temporal frequencies greater than 1 or $2 \mathrm{~Hz}$ ), motion aftereffects can be weak, but the spatial-frequency tuning is broad. ${ }^{43,44}$ These results are consistent with the suggestion that motion aftereffects experienced with static and dynamic test patterns reflect adaptation at different processing levels of the visual system with different tuning properties. ${ }^{43-46}$ Consistent with these data, some computational models of visual motion processing sum early (V1) inputs across spatial frequencies, resulting in broader spatial-frequency tuning at later (MT/V5) stages of processing. ${ }^{47,48}$

To reconcile the conflicting behavioral data, one must recognize that the stimuli employed have not been designed selectively to target the notional local or global motion processing mechanisms mentioned above (see Ref. 49 for a review). We have developed a paradigm for differentiating two motion-processing stages in human observers that has allowed us to make independent measures of the spatial-frequency selectivity of first-stage local motion detectors and second-stage global motion integrators. Three patterns of motion were examined-translation, rotation, and radiation (cardinal components of optic flow)-based on computational, ${ }^{50,51}$ behavioral, ${ }^{10}$ and electrophysiological ${ }^{19-22}$ studies implicating separate processing of these global patterns.

\section{RATIONALE}

\section{A. Local Motion Conditions}

Figure 1 shows a series of three element pairs. Each element is a dot that has been bandpass spatial-frequency filtered by an isotropic difference-of-Gaussian filter. The center frequencies of the elements in the left column are of medium spatial frequency, while the center frequency of the elements on the right includes medium, low, and 
high spatial frequencies. When either element is displaced (as suggested by the arrows), it will excite the receptive field of a local motion detector that is sensitive to the spatial frequency of the element. However, if the center frequency of the element is changed when it is displaced, the motion detector will respond only if it is sensitive to both the first and the second spatial frequency of the element-i.e., excitation will depend on the selectivity of the motion analyzer to the spatial frequencies of the two elements. Therefore the selectivity of the local motion detector can be determined from the change in motion sensitivity as a function of the change in spatial frequency of the first and the second dot. With this technique, a bandpass mechanism would be manifest by a loss in sensitivity when the spatial frequency of the second element is lower or higher than that of the first. A broadly tuned mechanism would respond roughly equally to all combinations of spatial frequencies, and sensitivity would be relatively unaffected by the spatial-frequency change.

(a) medium - medium

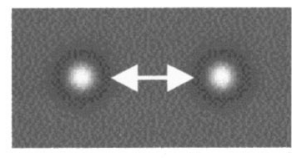

(b) medium - low

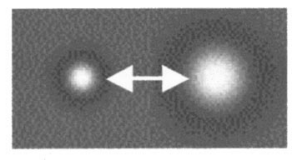

(c) medium - high

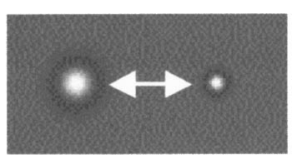

Fig. 1. Schematic illustration of the measurement of the spatial-frequency selectivity of local motion analyzers. Bilocal motion detectors respond only when the first and second instances of the element are within the spatial-frequency bandpass of the detector, e.g., diagram (a). Changes in motion sensitivity when the spatial frequency of one instance is (b) lower or (c) higher can be used to infer the selectivity of the unit. See the text for a detailed explanation.

\section{B. Global Motion Conditions}

Similar stimuli were employed to measure the spatialfrequency tuning of global motion detectors. As described in Subsection 2.A, the motion of the individual dot elements in random dot patterns is detected by local motion detectors. Because of the small receptive fields of these local detectors, ${ }^{1-4}$ the global pattern of these individual motions must be integrated across a large number of local detectors distributed across the stimulus. This process is illustrated schematically in Fig. 2. How similar must the local directional signals be in order to be integrated? For example, suppose that motion signals at different spatial scales are processed independently. In this case, the coherent motion of 4 -c/deg (c/deg $\equiv$ cycles per degree) signal elements would be unaffected by random motion of noise elements at 2 or $8 \mathrm{c} / \mathrm{deg}$, because these elements would excite a separate and independent population of motion detectors. Alternatively, suppose that motion signals at different scales are integrated by a mechanism that is sensitive to all spatial frequencies. In this case, the noise elements with a center frequency of 2 or $8 \mathrm{c} / \mathrm{deg}$ would mask the signal in the signal elements at $4 \mathrm{c} / \mathrm{deg}$ as effectively as in randomly moving elements at 4 c/deg. To test these competing predictions, we measured motion sensitivity for global motion patterns containing signal dots of the same spatial frequency moving in a coherent global pattern of motion (translation, rotation, or radiation) in the presence of noise elements of a range of spatial frequencies moving in random directions.

\section{METHODS}

\section{A. Apparatus}

Stimuli were generated on a Macintosh G3 computer with software adapted from the VideoToolbox routines ${ }^{52}$ and were displayed on a Sony PS400 monitor in gray scale at a frame rate of $75 \mathrm{~Hz}$. Stimuli had a mean luminance of $50 \mathrm{~cd} / \mathrm{m}^{2}$ and were displayed at $75 \%$ contrast. The luminance of the display was linearized with pseudo-12-bit resolution $^{53}$ in monochrome and calibrated with a Minolta photometer. Images were presented in gray scale by amplifying and sending the same 12-bit monochrome signal to all RGB guns of the display. The display mea-

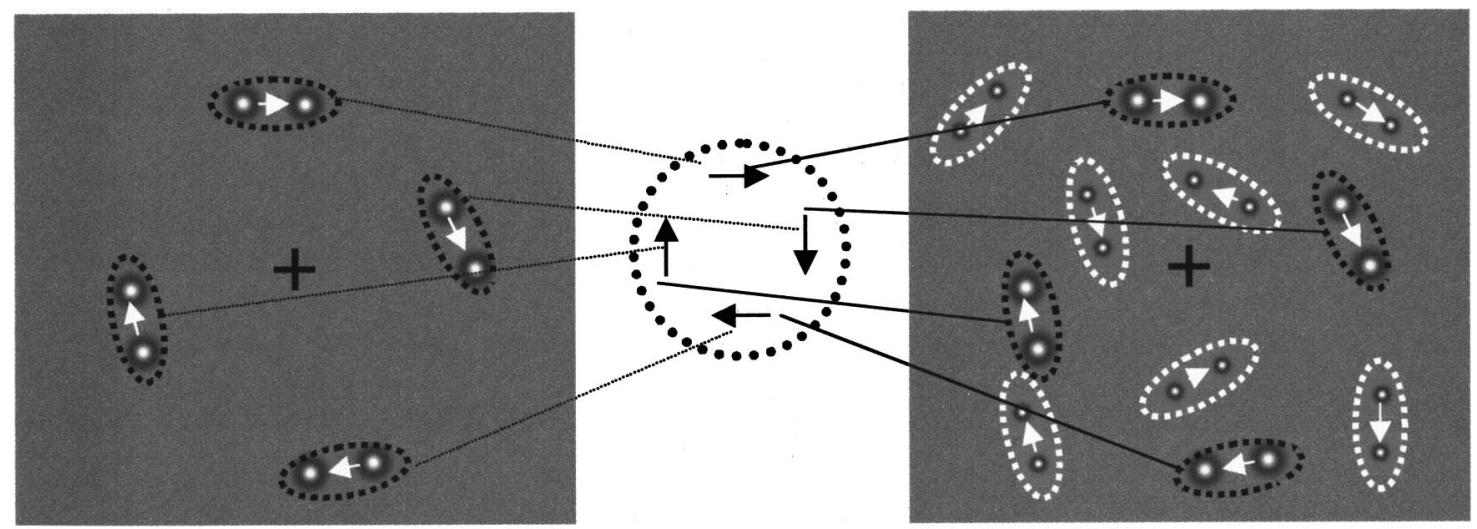

Fig. 2. Schematic illustration of the measurement of the spatial-frequency selectivity of global motion analyzers. The left image represents four local moving dots forming part of a global pattern of clockwise rotation. These local motions are integrated by a global motion detector, represented by the central collector unit. The right image shows the same four motions in the presence of noise elements (ringed in white) that move in random directions. The degree to which the noise elements of various spatial frequencies mask the coherent motion of the target elements can be used to infer the selectivity of the global unit. See the text for a detailed explanation. 
sured $20^{\circ}$ horizontally (832 pixels) and $15^{\circ}$ vertically (642 pixels) and was $57 \mathrm{~cm}$ from the observer in a dark room.

\section{B. Procedure}

Motion sensitivity was measured with a motion coherence paradigm. ${ }^{54}$ In this task, the elements in a field of random dots can move either coherently (termed signal dots) or in random directions (noise dots). Sensitivity to motion was determined by measuring the proportion of signal dots required for observers to discriminate on $75 \%$ of trials a pattern containing a proportion of elements moving in a coherent direction (e.g., leftward) from a pattern containing elements moving only in random directions. The proportion of signal dots on any trial was under computer control by means of an independent QUEST staircase. $^{55}$ Each QUEST was initialized with a random starting proportion of signal dots and concentrated observations about a signal level producing $75 \%$ correct responses. There were 32 trials for every run, and each run was completed a minimum of four times. Conditions were run in a random order. A cumulative normal was fitted to the combined data of the four runs by a least- $\chi^{2}$ procedure, which minimizes

$$
\sum\left(\frac{o_{i}-e_{i}}{\sigma_{i}}\right)^{2},
$$

where $o$ and $e$ represent the observed and expected proportion correct and $\sigma$ is the binomial standard deviation for a given level $i$. Motion coherence threshold and $95 \%$ confidence intervals were inferred from the $75 \%$ correct point of the psychometric function.

Subjects were presented with a series of two-interval forced-choice trials. One of the intervals (at random on each trial) contained a moving pattern in which all elements moved in random directions. In the other interval, a proportion of the elements were shifted in a coherent global pattern while the remainder moved in random directions. The observer's task was to fixate a central cross at all times and to indicate the interval containing coherent motion by pressing one of two mouse buttons. Auditory feedback was provided for incorrect responses. Three global patterns were tested on separate runs: translation (left/right), rotation (clockwise/anticlockwise), and radiation (expansion/contraction). Subjects were informed which global pattern was being tested on a given run. Motion coherence thresholds were defined as the proportion of signal dots required for observers to discriminate coherent motion from random motion on $75 \%$ of trials. Motion coherence sensitivity was defined as the inverse of motion coherence threshold.

\section{Stimuli}

Each animation frame contained 200 elements, where each element was a bandpass isotropic difference of Gaussians

$$
\begin{aligned}
L(x, y)= & {\left[\frac{1}{\sqrt{2 \pi} \sigma_{c}} \exp \left(-\frac{x^{2}}{2 \sigma_{c}^{2}}\right) \frac{1}{\sqrt{2 \pi} \sigma_{c}} \exp \left(-\frac{y^{2}}{2 \sigma_{c}^{2}}\right)\right] } \\
& -\left[\frac{1}{\sqrt{2 \pi} \sigma_{s}} \exp \left(-\frac{x^{2}}{2 \sigma_{s}^{2}}\right) \frac{1}{\sqrt{2 \pi} \sigma_{s}} \exp \left(-\frac{y^{2}}{2 \sigma_{s}^{2}}\right)\right],
\end{aligned}
$$

where $\sigma_{c}$ is the standard deviation of the center and $\sigma_{s}$ is the standard deviation of the surround Gaussian. The two Gaussians were dc balanced, and the standard deviation of the surround Gaussian was always 1.5 times that of the center Gaussian, giving a half-amplitude full bandwidth of 1.8 octaves.

The elements were shifted every third video frame to produce apparent motion. The displacement size of signal and noise dots depended on the condition. For rotation, the global rate of rotation was $0.5 \mathrm{cycle} / \mathrm{s}$, and the displacement size of signal and noise elements covaried with distance from the center. The displacement size of noise elements covaried with distance from the center in the same way as that for signal elements, but the direction of motion was random. Radial motion was produced by calculating the displacement as a function of eccentricity as for rotation at $0.5 \mathrm{cycle} / \mathrm{s}$, but the direction of movement was shifted by $90^{\circ}$ to produce a global pattern of expansion or contraction. The displacement of noise elements was the same as that for rotation. For translation, the displacement of signal and noise elements was 15 arc min, the direction of motion for signal elements was left or right, and the direction of motion of noise elements was random. This displacement was equal to that of elements at the middle of the radius for rotation and radial motion and was equivalent to a speed of $6 \mathrm{deg} / \mathrm{s}$ for continuous motion. The elements each had a "lifetime" of two frames (one displacement) to eliminate any coherent motion signal across alternate animation frames that could be tracked by observers. After it had been displaced, an element's life was over, it was randomly repositioned, and its lifetime was reset to 0 . Each element was initialized with a random starting age of 0 (in which case it was displaced by the required distance on the next frame) or 1 (in which case it was extinguished and repositioned on the next frame). This prevented the flicker that would occur if all elements expired simultaneously.

Five center frequencies were employed: 1, 1.4, 2, 2.8, and $4 \mathrm{c} / \mathrm{deg}$; illustrations of the stimuli are shown in Fig. 3. We set the displacement of the elements to be equal to ensure constant speeds for all center frequencies. However, this meant that elements of different center frequencies were displaced a different proportion of the peak frequency. In control conditions, we determined that motion coherence thresholds were equal for all center frequencies. Spatial antialiasing to 0.1-pixel accuracy was achieved by generating difference-of-Gaussian elements with 10-times magnification that were subsampled for each element drawn to each animation frame.

The elements were plotted within a circular aperture with a diameter subtending $8^{\circ}$ of visual angle. The outer edge of the circular aperture and the edge around the blank central fixation region were blurred with a raised cosine edge (extending 15 arc $\mathrm{min}$ ) to eliminate sharp edges at the display boundaries. Similarly, the onset and the offset of the stimuli were smoothed with a raised cosine temporal contrast envelope over $53 \mathrm{~ms}$.

Fourteen conditions were randomly interleaved on any run: five local and nine global motion conditions.

Local motion conditions: On each of the five local motion conditions, there were 200 moving elements. The computer manipulated the proportion of these elements 


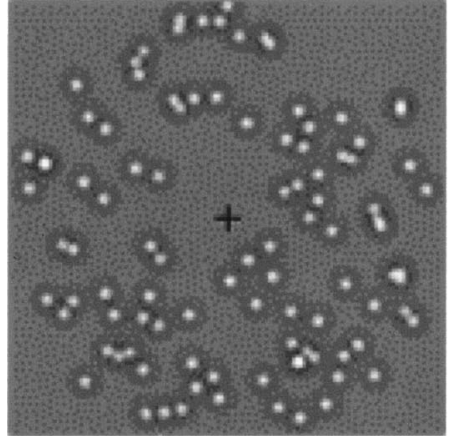

(a)

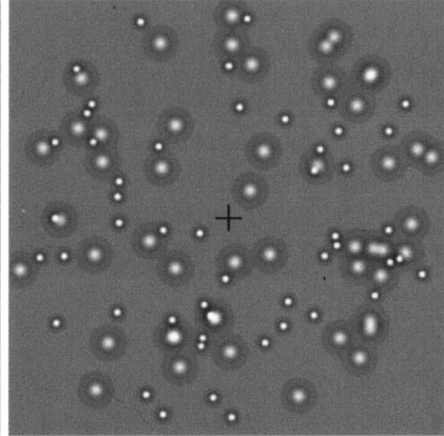

(b)

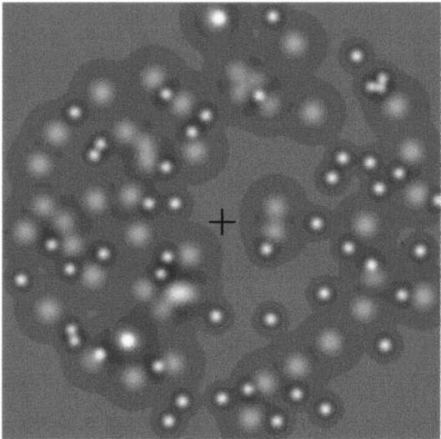

(c)

Fig. 3. Illustration of typical single frames from our movies, representing both local and global conditions: (a) 200 elements with the same peak spatial frequency (medium), (b) 100 elements at medium frequency and 100 at high frequency, (c) 100 elements at medium frequency and 100 at low frequency. Under local conditions, every element changed spatial frequency with each displacement. Under global conditions, target and noise elements did not change spatial frequency.

that moved in the signal direction (translation, rotation, or radiation); the direction of all remaining dots was selected at random. The spatial frequency of each element was swapped each time the element was displaced. The following pairings were employed: $1 \leftrightarrow 2,1.4 \leftrightarrow 2$, $2 \leftrightarrow 2,2.8 \leftrightarrow 2$, and $4 \leftrightarrow 2 \mathrm{c} / \mathrm{deg}$, where $1 \leftrightarrow 2$ means that every element changed spatial frequency between 1 and $2 \mathrm{c} / \mathrm{deg}$ with each displacement, and so on.

Global motion conditions. For the global motion conditions, stimuli were composed of 100 elements at the target spatial frequency and 100 elements at the mask spatial frequency. The computer manipulated the proportion of the target elements that moved in the signal direction (either a translation, a rotation, or a radiation, depending on the condition); the directions of the remaining elements at the target frequency were randomly assigned. All masking spatial elements moved in random directions. In five of the global motion conditions, the target spatial frequency was centered at $2 \mathrm{c} / \mathrm{deg}$, and the center frequency of mask elements was either 1, 1.4, 2, 2.8 , or $4 \mathrm{c} / \mathrm{deg}$. However, had we presented only these global conditions, it is possible that observers might have selectively attended only to the elements at $2 \mathrm{c} / \mathrm{deg}$ and tried to ignore the elements at other spatial frequencies (although, in practice, this is unlikely because local and global conditions were randomly interleaved). Nevertheless, to render this strategy ineffective, we included four additional global conditions in which the target spatial frequency was centered at either $1,1.4,2.8$, or $4 \mathrm{c} / \mathrm{deg}$, and the mask spatial frequency was fixed at $2 \mathrm{c} / \mathrm{deg}$. This made it impossible for observers to predict which elements carried the signal and which were noise, thus forcing them to consider elements at all spatial scales on every trial. Some previous investigations of motion perception employing a global motion coherence task have examined the effects of segmentation cues between signal and noise dots for translational motion. In some conditions, it has been reported that white signal dots are masked equally by black or white noise $\operatorname{dots}^{56,57}$; however, other studies have reported that observers can segment signal and noise dots by polarity and other cues, such as color $^{57,58}$ or depth. ${ }^{59,60}$ It has been argued that signal detection is facilitated only when observers can attend to the segmentation cues of the signal dots. ${ }^{60}$ Our paradigm effectively controls for such attentional effects.

\section{RESULTS AND DISCUSSION}

\section{A. Local Motion Detectors}

Figure 4 shows motion coherence sensitivity as a function of the fellow spatial frequency that was paired with a 2-c/ deg test element. Squares show data for global patterns of translation, circles show data for global patterns of radiation, and triangles show data for global patterns of rotation. The curves show the results of a least-squares fit to the data using a log-Gaussian function, weighted by the error bars. The data for the three global patterns were well fitted by log-Gaussian functions with the same peak frequency (PB: $2.02 \mathrm{c} / \mathrm{deg}, \mathrm{SD}: 1.96 \mathrm{c} / \mathrm{deg}$ ) and standard deviation (PB: 0.92 octave, SD: 1.04 octaves), as only a small reduction in residual was achieved by allowing these parameters to vary separately for each function. The functions were height shifted to allow for the differing sensitivities to the three classes of motion. It can be seen that motion coherence sensitivity falls precipitously with difference in center frequency. This finding is consistent with models that postulate narrowly tuned receptive fields for local motion detectors (e.g., Refs. 61-63).

\section{B. Global Motion Detectors}

Figure 5 shows motion coherence thresholds for two observers as a function of the spatial frequency of the masking elements. Squares show data for global patterns of translation, circles show data for global patterns of radiation, and triangles show data for global patterns of rotation. Solid symbols show thresholds for 100 elements at the target spatial frequency in the absence of any additional masking elements. The data have been leastsquares fitted with an exponential function, weighted by the error bars. The data for all global patterns of movement were fitted by exponential functions with the same decay constant (PB: $2.31 \mathrm{c} / \mathrm{deg}, \mathrm{SD}: 2.96 \mathrm{c} / \mathrm{deg}$ ), as only a small reduction in residual was achieved by allowing the decay constant to vary separately for each function. 
Again, the functions were height shifted to allow for the differing sensitivities to the three classes of motion. LogGaussian functions did not characterize the data as well but had large bandwidths (PB: 10.2 octaves, SD: 3.3 octaves). The data show that motion coherence thresholds were highest at the lowest mask center frequencies. This shows that whatever mechanism is pooling the motion of the signal elements is also sensitive to the low-frequency masking elements.

One potentially confounding variable in the global motion coherence task is that contrast sensitivity to the elements depends on the center frequency. It is therefore possible that the greater masking effect of low-frequency
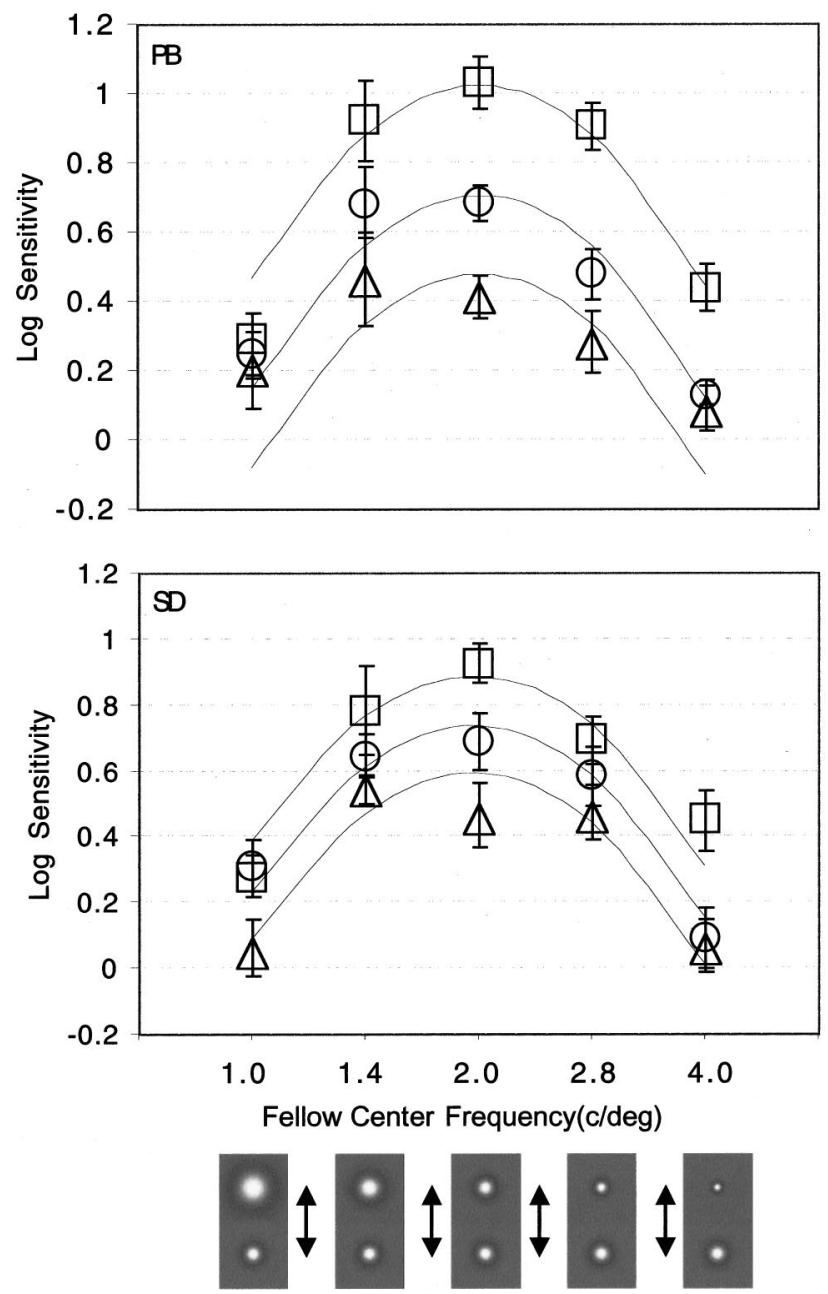

Fig. 4. Bandpass spatial-frequency selectivity of local motion analyzers. The plots show the results of experiment 1 for two observers (PB and SD) for global patterns of translation (squares), rotation (triangles), and radiation (circles). Each time that an element was displaced, its center frequency was swapped between a target frequency ( $2 \mathrm{c} / \mathrm{deg})$ and a fellow frequency $(1,1.4,2,2.8$, or $4 \mathrm{c} / \mathrm{deg})$, illustrated by the diagrams below the $x$ axis. Motion sensitivity (inverse of detection threshold) is shown as a function of center frequency of the fellow element. Error bars show 95\% confidence intervals. The data have been fitted with log-Gaussian functions (PB: $\mu=2.18 \mathrm{c} / \mathrm{deg}, \sigma=1.8$ octaves; SD: $\mu=1.96 \mathrm{c} / \mathrm{deg}, \sigma=1.8$ octaves). The functions have been vertically shifted because of the differing sensitivity to the three classes of motion (PB: radiation 2.2 times and rotation 3.7 times less sensitive than translation; SD: radiation 1.4 times and rotation 1.9 times less sensitive than translation).
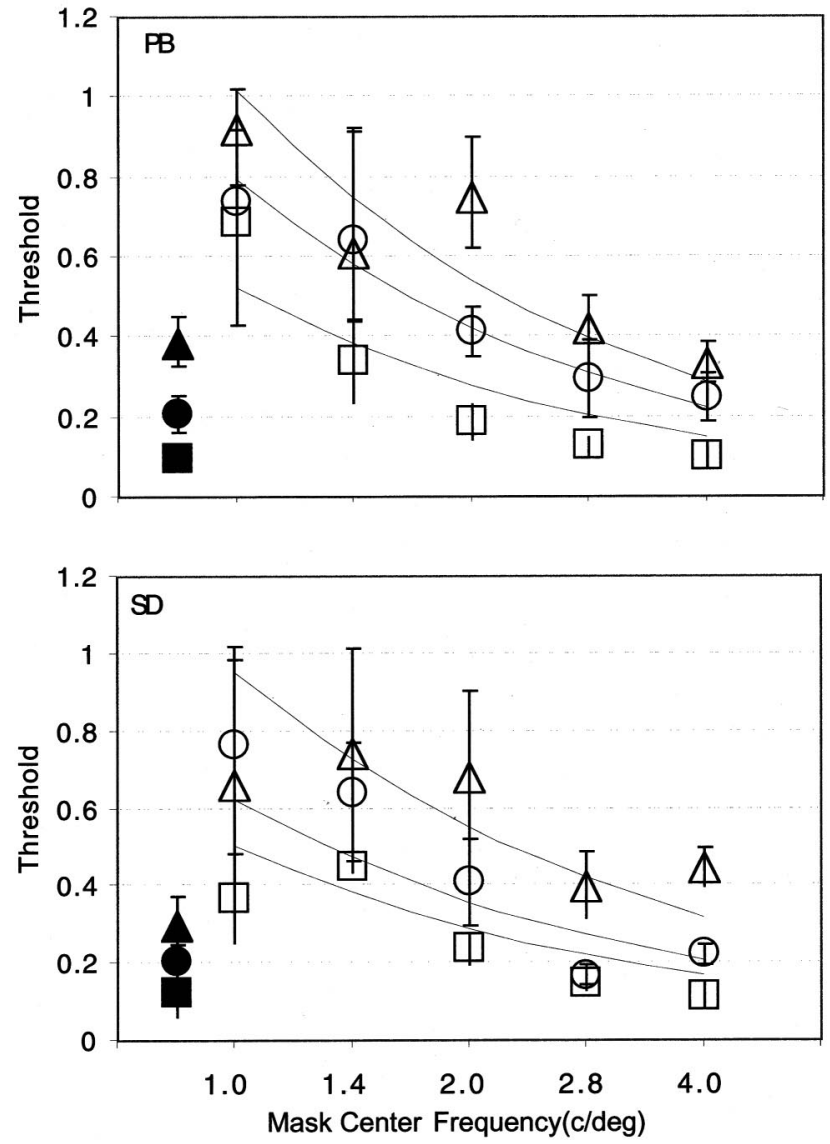

Fig. 5. Broadband spatial-frequency selectivity of global motion integrators. The plots show the results of experiment 2 for two observers (PB and SD) for global patterns of translation (squares), rotation (triangles), and radiation (circles). Motion coherence thresholds were measured for 100 elements of target center frequency $(2 \mathrm{c} / \mathrm{deg})$ in the presence of 100 masking elements of fellow center frequency $(1,1.4,2,2.8$, or $4 \mathrm{c} / \mathrm{deg})$, illustrated by the diagrams below the $x$ axis. The filled symbols on the left show detection thresholds for 100 elements at the target frequency $(2 \mathrm{c} / \mathrm{deg})$ with no masking elements present. Motion coherence thresholds are shown as a function of fellow center frequency. Error bars show 95\% confidence intervals. The data have been fitted with exponential functions (PB: $t=2.31$, SD: $t=2.95)$. The functions have been vertically shifted because of the differing sensitivity to the three classes of motion (PB: radiation 1.5 times and rotation 1.9 times less sensitive than translation; SD: radiation 1.7 times and rotation 2.1 times less sensitive than translation).

elements reported in experiment 2 could simply be the result of higher sensitivity to low-spatial-frequency elements. This explanation is unable to explain the pattern of results in experiment 1 , because motion sensitivity fell whether the fellow spatial frequency was lower or higher than that of the target. Although it has been shown that apparent contrast is independent of spatial frequency at high contrasts, ${ }^{64}$ we repeated experiment 2 with elements at a contrast that was 5 times their contrast detection threshold. The procedure for measuring contrast detection thresholds was the same as that in the main experiment, except that observers were required to discriminate an interval containing 200 translating elements at 50\% coherence from a blank interval, with auditory feedback provided on incorrect trials. Contrast thresholds for 
translation, rotation, and radiation were compared for one observer (PB) and were the same for each global pattern. The results of the global motion sensitivity task with all elements at 5 times contrast threshold are shown in Fig. 6. It can be seen that in this case the motion of the target elements was effectively masked by all spatial frequencies. Thus global motion integrators are sensitive to a broad range of spatial frequencies if the contrast of the elements is sufficiently high.

Contribution of second-order motion detector. An alternative explanation of the broad spatial-frequency tuning of global motion detectors could be based on the operation of second-order ${ }^{65}$ or perhaps third-order 66 / feature-tracking ${ }^{67}$ motion detectors. Following full- or half-wave rectification, ${ }^{65,68,69}$ second-order motion detectors would respond to the circular envelope of our stimuli, which, although of different sizes, contain common lowspatial-frequency structure after rectification. It is therefore possible that the broad spatial-frequency tuning that we observe under global motion conditions could simply reflect the activity of second-order motion detectors
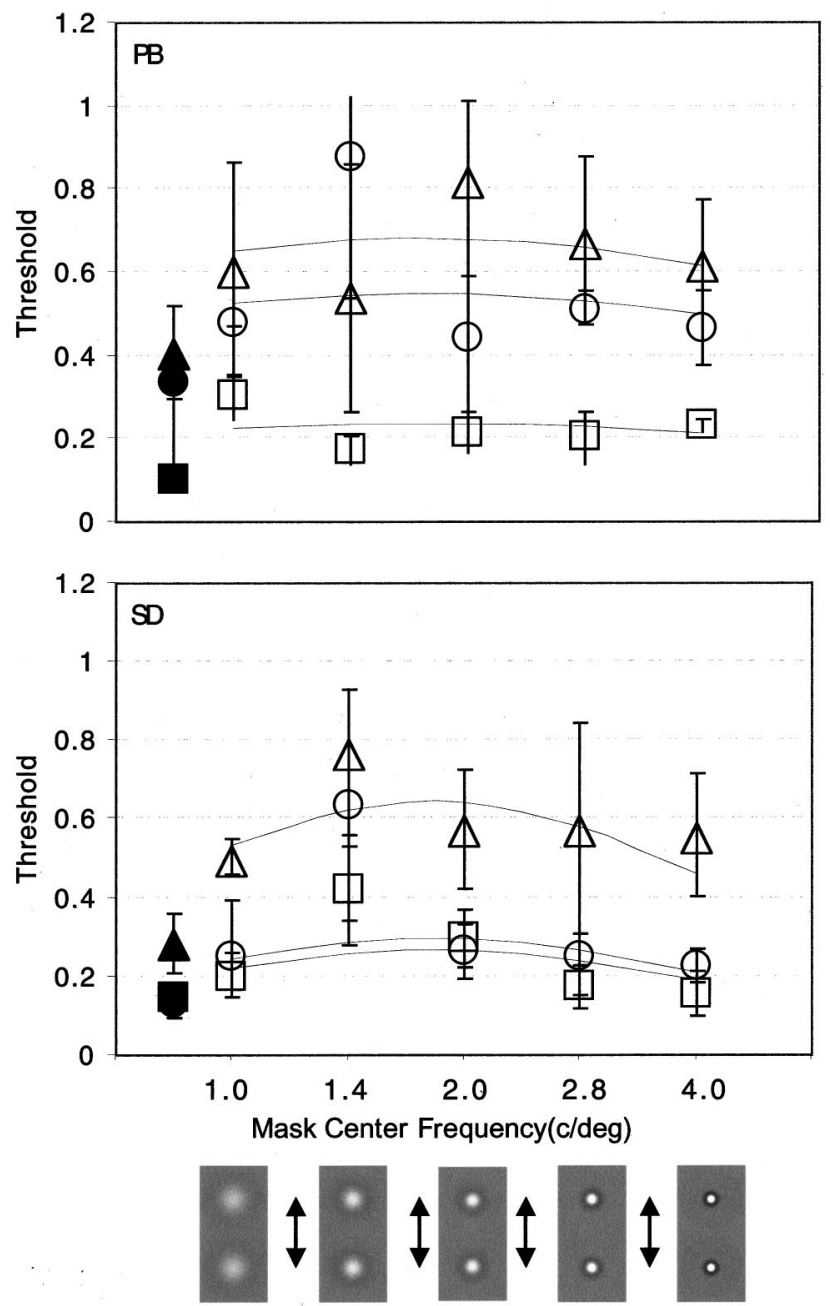

Fig. 6. Broadband spatial-frequency selectivity of global motion integrators. The results are the same as those in Fig. 5, except that the contrast of each element was adjusted for contrast sensitivity to be 5 times the detection threshold for each spatial frequency and the curves show the best-fitting log-Gaussian functions. responding to this structure. Similarly, featuretracking ${ }^{66,67,70}$ processes could, in principle, track motion between elements of differing center frequency, resulting in little or no spatial-frequency tuning. However, the limited lifetime, the brief exposure duration, the high density, and the stochastic properties of our stimuli are not favourable for second-order ${ }^{71-73}$ or featuretracking ${ }^{66,74}$ mechanisms, casting doubt on this explanation. Moreover, a further factor militates against this view. The nonlinear processing of second- and thirdorder detectors would be expected to occur under both our local and global motion conditions (the conditions were all interleaved, so the observer had no way of knowing whether the trial was a local or a global condition). One would therefore have expected broad spatial-frequency tuning under both conditions, however, the tuning that we observe is clearly different for each condition.

\section{CONCLUSIONS}

The results support behavioral and physiological studies showing that global motion perception involves two stages of analysis. Here we report that first-stage local motion analyzers are narrowly tuned for spatial frequency, while second-stage global motion detectors are broadly tuned for spatial frequency. These results reconcile puzzling differences in spatial-frequency selectivity found in previous studies implicating narrow ${ }^{36,37,40-42}$ or broad $^{39,43,44}$ spatial-frequency selectivity for motion perception.

\section{ACKNOWLEDGMENTS}

S. C. Dakin was supported by the Wellcome Trust. We thank two anonymous reviewers for helpful comments.

Corresponding author Peter Bex's e-mail address is p.bex@ucl.ac.uk.

\section{REFERENCES}

1. D. H. Hubel and T. N. Wiesel, "Receptive fields and functional architecture of monkey striate cortex," J. Physiol. (London) 195, 215-243 (1968).

2. R. H. Wurtz, "Visual receptive fields of striate cortex neurons in awake monkeys," J. Neurophysiol. 32, 727-742 (1969).

3. P. H. Schiller, B. L. Finlay, and S. F. Volman, "Quantitative studies of single-cell properties in monkey striate cortex. I. Spatiotemporal organization of receptive fields," J. Neurophysiol. 39, 1288-1399 (1976).

4. S. J. Anderson and D. C. Burr, "Receptive field size of human motion detection units," Vision Res. 27, 621-635 (1987).

5. P. Verghese and L. S. Stone, "Combining speed information across space," Vision Res. 35, 2811-2823 (1995).

6. P. Verghese and L. S. Stone, "Perceived visual speed constrained by image segmentation," Nature (London) 381, 161-163 (1996).

7. D. Regan and K. I. Beverly, "Looming detectors in the human visual pathway," Vision Res. 18, 415-421 (1978). 
8. T. C. A. Freeman and M. G. Harris, "Human sensitivity to expanding and rotating motion: effects of complementary masking and directional structure," Vision Res. 32, 81-87 (1992).

9. M. C. Morrone, D. C. Burr, and L. M. Vaina, "Two stages of visual processing for radial and circular motion," Nature (London) 376, 507-509 (1995)

10. M. C. Morrone, D. C. Burr, and S. Di Pietro, "Cardinal directions for visual optic flow," Curr. Biol. 9, 763-766 (1999).

11. D. C. Burr, M. C. Morrone, and L. M. Vaina, "Large receptive fields for optic flow detection in humans," Vision Res. 38, 1731-1743 (1998)

12. K. Gurney and M. J. Wright, "Rotation and radial motion thresholds support a two-stage model of differential-motion analysis," Perception 25, 5-26 (1996).

13. M. Lappe and J. P. Rauschecker, "An illusory transformation in a model of optic flow processing," Vision Res. 35 1619-1631 (1995).

14. R. J. Snowden and A. B. Milne, "Phantom motion aftereffects-evidence of detectors for the analysis of optic flow," Curr. Biol. 7, 717-722 (1997).

15. P. J. Bex, A. B. Metha, and W. Makous, "Psychophysical evidence for a functional hierarchy of motion processing mechanisms," J. Opt. Soc. Am. A 15, 769-776 (1998).

16. P. J. Bex and W. Makous, "Radial motion looks faster," Vision Res. 37, 3399-3405 (1997).

17. P. J. Bex, A. B. Metha, and W. Makous, "Enhanced motion aftereffect for complex motions," Vision Res. 39, 2229-2238 (1999).

18. H. A. Saito, K. Tanaka, H. Isono, M. Yasuda, and A. Mikami, "Integration of direction signals of image motion in the superior temporal sulcus of the macaque monkey," J. Neurosci. 61, 145-157 (1986).

19. C. J. Duffy and R. H. Wurtz, "Sensitivity of MST neurons to optic flow stimuli. I. A continuum of response selectivity to large-field stimuli,” J. Neurophysiol. 65, 1329-1345 (1991).

20. G. A. Orban, L. Lagae, A. Verri, S. Raiguel, D. Xiao, H. Maes, and V. Torre, "First-order analysis of optical flow in monkey brain,” Proc. Natl. Acad. Sci. USA 89, 2595-2599 (1992).

21. K. Tanaka and H. Saito, "Analysis of motion of the visual field by direction, expansion/contraction, and rotation cells clustered in the dorsal part of the medial superior temporal area of the macaque monkey," J. Neurophysiol. 62, 626-641 (1989).

22. M. S. Graziano, R. A. Andersen, and R. J. Snowden, "Tuning of MST neurons to spiral motions," J. Neurosci. 14, 54-67 (1994).

23. J. Kim, K. Mulligan, and H. Sherk, "Simulated optic flow and extrastriate cortex. I: optic flow versus texture," J. Neurophysiol. 77, 554-561 (1997).

24. K. Mulligan, J. Kim, and H. Sherk, "Simulated optic flow and extrastriate cortex. II: responses to bar versus largefield stimuli," J. Neurophysiol. 77, 562-570 (1997).

25. H. G. Krapp and R. Hengstenberg, "Estimation of self motion by optic flow processing in single visual interneurons," Nature (London) 384, 463-466 (1996).

26. O. J. Braddick, "A short-range process in apparent motion," Vision Res. 14, 519-527 (1974)

27. J. J. Chang and B. Julesz, "Displacement limits for spatial frequency filtered random dot cinematograms in apparent motion," Vision Res. 23, 1379-1385 (1983).

28. J. J. Chang and B. Julesz, "Cooperative and noncooperative processes of apparent movement of random-dot cinematograms," Spatial Vision 1, 39-45 (1985).

29. R. Cleary and O. J. Braddick, "Masking of low frequency information in short-range apparent motion," Vision Res. 30, 317-327 (1990).

30. R. Cleary and O. J. Braddick, "Direction discrimination for band-pass filtered random dot kinematograms," Vision Res. 30, 303-316 (1990).

31. W. F. Bischof and V. Di Lollo, "Perception of directional sampled motion in relation to displacement and spatial frequency: evidence for a unitary motion system," Vision Res. 30, 1341-1362 (1990).
32. M. J. Morgan and G. Mather, "Motion discrimination in two-frame sequences with differing spatial frequency content," Vision Res. 34, 197-208 (1994).

33. P. J. Bex, N. Brady, R. E. Fredericksen, and R. F. Hess, "Energetic motion detection," Nature (London) 378, 670-672 (1995).

34. R. A. Eagle and B. J. Rogers, "Motion detection is limited by element density not spatial frequency," Vision Res. 36, 545558 (1996)

35. N. Brady, P. J. Bex, and R. E. Fredericksen, "Independent coding across spatial scales in moving fractal images," Vision Res. 37, 1873-1883 (1997).

36. T. Ledgeway, "How similar must the Fourier spectra of the frames of a random-dot kinematogram be to support motion perception?" Vision Res. 36, 2489-2495 (1996).

37. A. B. Watson, "Apparent motion occurs only between similar spatial frequencies," Vision Res. 26, 1727-1730 (1986).

38. R. F. Hess, P. J. Bex, R. F. Fredericksen, and N. Brady, "Is human motion detection subserved by a single or multiple channel mechanism?" Vision Res. 38, 259-266 (1998).

39. Y. D. Yang and R. Blake, "Broad tuning for spatialfrequency of neural mechanisms underlying visualperception of coherent motion," Nature (London) 371, 793796 (1994).

40. R. Over, J. Broerse, B. Crassini, and W. Lovegrove, "Spatial determinants of the aftereffect of seen movement," Vision Res. 13, 1681-1690 (1973)

41. E. L. Cameron, C. L. Baker, and J. C. Boulton, "Spatial frequency selective mechanisms underlying the motion aftereffect," Vision Res. 32, 561-568 (1992).

42. P. J. Bex, F. A. Verstraten, and I. Mareschal, "Temporal and spatial frequency tuning of the flicker motion aftereffect," Vision Res. 36, 2721-2727 (1996).

43. I. Mareschal, H. Ashida, P. J. Bex, S. Nishida, and F. A. J. Verstraten, "Temporal frequency tuning of the test pattern: the missing link between lower and higher stages of motion processing as revealed by the flicker motion aftereffect?" Vision Res. 37, 1755-1759 (1997)

44. H. Ashida and N. Osaka, "Difference of spatial-frequency selectivity between static and flicker motion aftereffects," Perception 23, 1313-1320 (1994).

45. F. A. J. Verstraten, R. E. Fredericksen, R. J. A. van Wezel, M. J. M. Lankheet, and W. A. van de Grind, "Recovery from adaptation for dynamic and static motion aftereffects: evidence for two mechanisms," Vision Res. 36, 421-424 (1996).

46. S. Nishida and T. Sato, "Motion aftereffect with flickering test patterns reveals higher stages of motion processing," Vision Res. 35, 477-490 (1995).

47. D. J. Heeger, "Model for the extraction of image flow," J. Opt. Soc. Am. A 4, 1455-1471 (1987).

48. E. P. Simoncelli and D. J. Heeger, "A model of neuronal responses in visual area MT," Vision Res. 38, 743-761 (1998).

49. O. Braddick, "Segmentation versus integration in visual motion processing," Trends Neurosci. 16, 263-268 (1993).

50. J. J. Koenderink, "Optic flow," Vision Res. 26, 161-179 (1986).

51. J. J. Koenderink and A. J. van Doorn, "How an ambulant observer can construct a model of the environment from the geometrical structure of the visual inflow," in Kibernetic, G. Hauske and E. Butendant, eds. (Oldenbourg, Munich, 1977).

52. D. G. Pelli, "The VideoToolbox software for visual psychophysics: transforming numbers into movies," Spatial Vision 10, 437-442 (1997).

53. D. G. Pelli and L. Zhang, "Accurate control of contrast on microcomputer displays," Vision Res. 31, 1337-1350 (1991).

54. W. T. Newsome and E. B. Pare, "A selective impairment of motion perception following lesions of the middle temporal visual area (MT)," J. Neurosci. 8, 2201-2211 (1988).

55. A. B. Watson and D. G. Pelli, "QUEST: a Bayesian adaptive psychometric method," Percept. Psychophys. 33, 113120 (1983).

56. M. Edwards and D. R. Badcock, "Interactions of the $\mathrm{ON}$ and OFF pathway," Vision Res. 34, 2849-2858 (1994). 
57. R. J. Snowden and R. Edmunds, "Colour and polarity contributions to global motion perception," Vision Res. 39, 1813-1822 (1999).

58. L. J. Croner and T. D. Albright, "Image segmentation enhances discrimination of motion in visual noise," Vision Res. 37, 1415-1427 (1997).

59. P. B. Hibbard, M. F. Bradshaw, and B. De Bruyn, "Is global motion tuned for binocular disparity?" Vision Res. 39, 961974 (1999).

60. R. J. Snowden and M. C. Rossiter, "Stereoscopic depth cues can segment motion information," Perception 28, 193-201 (1999).

61. E. H. Adelson and J. R. Bergen, "Spatiotemporal energy models for the perception of motion," J. Opt. Soc. Am. A 2, 284-299 (1985).

62. A. B. Watson and A. J. Ahumada, Jr., "Model of human visual-motion sensing," J. Opt. Soc. Am. A 2, 322-342 (1985).

63. J. P. van Santen and G. Sperling, "Elaborated Reichardt detectors," J. Opt. Soc. Am. A 2, 300-321 (1985).

64. M. A. Georgeson and G. D. Sullivan, "Contrast constancy: deblurring in human vision by spatial frequency channels," J. Physiol. (London) 252, 627-656 (1975).

65. C. Chubb and G. Sperling, "Drift-balanced random stimuli: a general basis for studying non-Fourier motion perception," J. Opt. Soc. Am. A 5, 1986-2006 (1988).
66. Z. Lu and G. Sperling, "The functional architecture of human visual motion perception," Vision Res. 35, 2697-2722 (1995).

67. S. Ullman, The Interpretation of Visual Motion (MIT Press, Cambridge, Mass., 1979).

68. H. R. Wilson, V. P. Ferrera, and C. Yo, "A psychophysically motivated model for two-dimensional motion perception," Visual Neurosci. 9, 79-97 (1992).

69. P. Werkhoven, G. Sperling, and C. Chubb, "The dimensionality of texture defined motion: a single channel theory," Vision Res. 33, 463-485 (1993).

70. P. Cavanagh, M. Arguin, and M. von Grunau, "Interattribute apparent motion," Vision Res. 29, 1197-1204 (1989).

71. J. C. Boulton, and C. L. Baker, Jr., "Different parameters control motion perception above and below a critical density," Vision Res. 33, 1803-1811 (1993).

72. J. C. Boulton and C. L. Baker, "Dependence on stimulus onset asynchrony in apparent motion: evidence for two mechanisms," Vision Res. 33, 2013-2019 (1993).

73. C. L. J. Baker and R. F. Hess, "Two mechanisms underlie processing of stochastic motion stimuli," Vision Res. 38, 1211-1222 (1998).

74. P. J. Bex and C. L. Baker, "Motion perception over long inter-stimulus intervals," Percept. Psychophys. 61, 10661074 (1999). 